\title{
Interpretation of the LHC run-1 Higgs results (2HDM)
}

\author{
Rui Santos ${ }^{* \dagger}$ \\ Instituto Superior de Engenharia de Lisboa - ISEL, 1959-007 Lisboa, Portugal and \\ Centro de Física Teórica e Computacional, Faculdade de Ciências, Universidade de Lisboa, \\ Av. Prof. Gama Pinto 2, 1649-003 Lisboa, Portugal \\ E-mail: rasantos@fcul.pt
}

\begin{abstract}
We present the status of the CP-conserving two-Higgs doublet model at the end of the $8 \mathrm{TeV}$ run. We discuss how far can one go in probing the wrong sign limit and the symmetric limit of the $2 \mathrm{HDM}$ during the next LHC run. The heavy scenario, defined as the one where the heavy CP even scalar is the one discovered at the LHC, has no decoupling limit. This non-decoupling nature of the heavy scenario is extremely interesting and there are good chances to probe it at the next LHC run.
\end{abstract}

Prospects for Charged Higgs Discovery at Colliders

16-18 September 2014

Uppsala University Sweden

*Speaker.

$\dagger$ I thank the conference organizers for the invitation and for the nice, familiar and productive atmosphere. I also thank the participants' warm welcome of MJ Santos in her conference début. I acknowledge financial support from the conference organizers and from FCT under contracts PTDC/FIS/117951/2010 and PEst-OE/FIS/UI0618/2011. I thank JP Silva for reading the manuscript. 


\section{Introduction}

The discovery of the Higgs bosons at the Large Hadron Collider by the ATLAS [1] and CMS [2] collaborations was undoubtedly a major achievement in science. Now that all run-1 data has been analysed it is clear that the Higgs boson resembles the one predicted by the Standard Model (SM). In fact, the measurement of Higgs production cross sections and branching ratios clearly point to a very SM-like Higgs boson. It is now the time to understand if extensions of the SM, that can also explain the experimental results, can be probed at the next LHC run. Many SM extensions provide a number of scenarios that although allowed at the end of run-1, could be probed/excluded during the next LHC run.

We will focus on one of the simplest extensions of the SM, the two-Higgs doublet model (2HDM), which is build by simply adding one extra scalar doublet to the SM field content (for a review see [3, 4]). The $2 \mathrm{HDM}$ comes in many different forms. There are CP-conserving versions, CP-breaking versions and it can also provide a dark matter candidate. We will start by reviewing the most general 2HDM Yukawa sector followed by a brief presentation of the Higgs potential. We will then move to the main purpose of this work which is the interpretation of the results from run-1 in the context of the CP-conserving 2HDM. We will analyse the different scenarios that can be probed at the next LHC run. Finally we will briefly comment on other versions of the 2HDM.

\section{Yukawa Lagrangian and Flavour Changing Neutral Currents}

The SM Yukawa Lagrangian can be written as

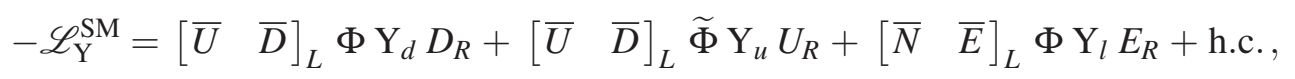

where $U, D, N$ and $E$ are gauge eigenstates vectors in flavour space for up-type quarks, downtype quarks, neutrinos and charged leptons, respectively and $\mathrm{Y}$ are $3 \times 3$ matrices in flavour space. Defining the vacuum expectation value of the neutral component of the Higgs doublet $\Phi^{0}$ as $\left\langle\Phi^{0}\right\rangle=$ $v / \sqrt{2}$, the mass terms are

$$
-\mathscr{L}_{\mathrm{Y}}^{\mathrm{SM}(\mathrm{mass})}=\frac{v}{\sqrt{2}}\left(\bar{U}_{L} \mathrm{Y}_{u} U_{R}+\bar{D}_{L} \mathrm{Y}_{d} D_{R}+\bar{E}_{L} \mathrm{Y}_{e} E_{R}+\text { h.c. }\right) .
$$

Let us focus, for simplicity, on the down-type quarks only ${ }^{1}$. Defining the rotation to the mass eigenstates as $D_{R} \rightarrow N_{R} D_{R}$ and $D_{L} \rightarrow N_{L} D_{L}$, leads to a mass matrix for down-type quarks $\mathrm{M}_{d}=$ $-v / \sqrt{2}\left(N_{L}^{\dagger} \mathrm{Y}_{d} N_{R}\right)+$ h.c.. It is straightforward to show that the Higgs interaction with downtype quarks is $h \bar{D}_{L} \mathrm{M}_{d} D_{R}+$ h.c.. Hence, there are no tree-level flavour changing neutral currents (FCNC) in the SM. This is obviously related to the proportionality between the interaction and the mass term in the initial Lagrangian, that is $h \bar{D}_{L} \mathrm{Y}_{d} D_{R} \propto v \bar{D}_{L} \mathrm{Y}_{d} D_{R}$.

In the 2HDM, there are now two doublets, $\Phi_{1}$ and $\Phi_{2}$. The real and neutral components of the gauge eigenstates are now defined as $h_{1}$ and $h_{2}$ with real VEVs $\left\langle\Phi_{1}^{0}\right\rangle=v_{1} / \sqrt{2}$ and $\left\langle\Phi_{2}^{0}\right\rangle=v_{2} / \sqrt{2}$. The most general Yukawa Lagrangian is now

$$
-\mathscr{L}_{\mathrm{Y}}^{2 \mathrm{HDM}}=\sum_{a=1}^{2}\left[\begin{array}{ll}
\bar{U} & \bar{D}
\end{array}\right]_{L} \Phi_{a} \mathrm{Y}_{d}^{a} D_{R}+\left[\begin{array}{ll}
\bar{U} & \bar{D}
\end{array}\right]_{L} \widetilde{\Phi}_{a} \mathrm{Y}_{u}^{a} U_{R}+\left[\begin{array}{ll}
\bar{N} & \bar{E}
\end{array}\right]_{L} \Phi_{a} \mathrm{Y}_{l}^{a} E_{R}+\text { h.c. },
$$

\footnotetext{
${ }^{1}$ Since we are considering massless neutrinos, in the lepton sector $E$ can be chosen as the mass eigenstates vector.
} 
Focusing again on the down-type quarks only, the mass lagrangian is

$$
-\mathscr{L}_{\mathrm{Y}}^{2 \mathrm{HDM}(\text { mass })}=\frac{1}{\sqrt{2}}\left(\bar{D}_{L}\left(v_{1} \mathrm{Y}_{d}^{1}+v_{2} \mathrm{Y}_{d}^{2}\right) D_{R}+\text { h.c. }\right) \ldots,
$$

while the corresponding interaction terms are

$$
-\mathscr{L}_{\mathrm{Y}}^{2 \mathrm{HDM}}=\frac{H}{\sqrt{2}}\left(\bar{D}_{L}\left(-\sin \alpha \mathrm{Y}_{d}^{1}+\cos \alpha \mathrm{Y}_{d}^{2}\right) D_{R}+\text { h.c. }\right) \ldots,
$$

where the mass eigenstate $H$ is obtained from $h_{1}$ and $h_{2}$ via $H=-\sin \alpha h_{1}+\cos \alpha h_{2}$. There is a similar term for the other mass eigenstate $h=\cos \alpha h_{1}+\sin \alpha h_{2}$ for which the down-type quark coupling is proportional to $\left(-\sin \alpha \mathrm{Y}_{d}^{1}+\cos \alpha \mathrm{Y}_{d}^{2}\right)$. Clearly the mass terms is not proportional to any of the Higgs interaction terms. Therefore they cannot be diagonalised simultaneously which leads, in the most general case, to tree-level FCNC. It is well known that these FCNC mediated by scalars are severely constrained by low-energy experiments.

The issue of avoiding FCNCs has been addressed in many different ways over the years. The simplest solution is to couple fermions of a given electric charge to no more than one Higgs doublet [5]. This can be accomplished in a natural way by imposing a $\mathbb{Z}_{2}$ symmetry to all fields and invariance of the lagrangian under that symmetry. The $\mathbb{Z}_{2}$ charge assignments $\left(\Phi_{1}\right.$ is even and $\Phi_{2}$ is odd) lead to four independent combinations [6]: only $\Phi_{2}$ couples to all fermions (type I); $\Phi_{2}$ couples to up-type quarks while $\Phi_{1}$ couples to charged leptons and down-type quarks (type II); $\Phi_{2}$ couples to charged leptons and up-type quarks while $\Phi_{1}$ couples to down-type quarks (type Flipped or Y); $\Phi_{2}$ couples to quarks while $\Phi_{1}$ couples to charged leptons (type Lepton Specific or X). One of the doublets is odd while the other is even under $\mathbb{Z}_{2}$.

All other versions of 2HDMs where this symmetry is not imposed in the Yukawa sector either have tree-level FCNC or are not stable under the renormalization group [7] (see [4] for a thorough discussion). There are however ways to force those flavour changing currents to be small. One such example is a class of models known as BGL [8] where the tree-level flavour changing couplings are proportional to the elements of the CKM matrix. Therefore the off-diagonal CKM elements naturally suppress the neutral scalars flavour changing couplings. Another example is the ChengSher Ansatz [9] where the flavour-changing couplings are proportional to the geometric mean of the Yukawa couplings of the two fermions involved in coupling.

There are other proposals in the literature for 2HDMs where FCNC is absent at tree-level. One such example is the Aligned model [10] which assumes that the two Yukawa-coupling matrices are proportional precluding the existence of tree-level FCNC. Although these type of models can provide an interesting guide for phenomenological purposes they are however not stable under renormalization [7].

\section{2HDM Higgs potential}

Once we have chosen to avoid FCNC at tree-level by imposing the $\mathbb{Z}_{2}$ symmetry on the fields, the Higgs potential invariant under $\mathbb{Z}_{2}$, softly broken by a dimension two term, can be written as

$$
\begin{gathered}
\mathscr{V}=m_{11}^{2} \Phi_{1}^{\dagger} \Phi_{1}+m_{22}^{2} \Phi_{2}^{\dagger} \Phi_{2}-\left(m_{12}^{2} \Phi_{1}^{\dagger} \Phi_{2}+\text { h.c. }\right)+\frac{1}{2} \lambda_{1}\left(\Phi_{1}^{\dagger} \Phi_{1}\right)^{2}+\frac{1}{2} \lambda_{2}\left(\Phi_{2}^{\dagger} \Phi_{2}\right)^{2} \\
+\lambda_{3} \Phi_{1}^{\dagger} \Phi_{1} \Phi_{2}^{\dagger} \Phi_{2}+\lambda_{4} \Phi_{1}^{\dagger} \Phi_{2} \Phi_{2}^{\dagger} \Phi_{1}+\left[\frac{1}{2} \lambda_{5}\left(\Phi_{1}^{\dagger} \Phi_{2}\right)^{2}+\text { h.c. }\right] .
\end{gathered}
$$


Since $\mathscr{V}$ has to be hermitian, $m_{12}^{2}$ and $\lambda_{5}$ can be complex while all other parameters have to be real. If $m_{12}^{2}, \lambda_{5}$ and both VEVs are real, the potential is CP-conserving. An explicit CP-violating potential is obtained by choosing $m_{12}^{2}$ and $\lambda_{5}$ complex [11]. Since the VEVs are real we can define $\tan \beta=v_{2} / v_{1}$ for both models. The remaining independent parameters for the CP-conserving model are the four masses $m_{h}, m_{H}, m_{A}$ and $m_{H^{ \pm}}$, the rotation angle $\alpha$ that diagonalises the CP-even mass matrix, and $m_{12}^{2}$. In the CP-violating case the free parameters can be chosen as the two lighter neutral states masses $m_{1}$ and $m_{2}, m_{H^{ \pm}}$, the three rotation angles that diagonalise the neutral mass matrix $\alpha_{1}, \alpha_{2}$ and $\alpha_{3}$ and $\operatorname{Re}\left[\mathrm{m}_{12}^{2}\right]$.

Once a CP-conserving vacuum configuration is chosen, it is stable against the appearance of both charge breaking and CP-breaking minima, at tree-level [12]. It is however possible that up to two CP-conserving minima coexist. However, we force the $\mathrm{CP}$-conserving minimum with the right pattern of symmetry breaking to be the global one [13].

\section{The status of the CP-conserving 2HDM}

The constraints on the CP-conserving 2HDM were recently reviewed in [14] and are included either directly or via interface with other codes in SCANNERS [15, 16], which was used to perform all scans over the 2HDM parameter space. SCANNERS is interfaced with SUSHI [17] which calculates $p p(g g+b b) \rightarrow h$ at NNLO and with HDECAY $[18,19]$ for the calculation of all Higgs decays. All other production cross sections, vector boson fusion (VBF), associated production (with a $W$ or a $Z$ ) and $t \bar{t} h$ were taken from [20] at NLO. All collider data was taken into account via interface with external codes. The non-observation of new scalars was considered using HiggsBounds [21] with the exclusion limits taken at $95 \%$ C.L.. Consistency within a $3 \sigma, 2 \sigma$ or $1 \sigma$ probability with the observed Higgs boson at the LHC was taken into account with HigGSSignals [22].

When confronting the $2 \mathrm{HDM}$ with the Higgs experimental results obtained at $8 \mathrm{TeV}$ we use the signal strength defined as

$$
\mu_{f}^{h}=\frac{\sigma \mathrm{BR}(h \rightarrow f)}{\sigma^{\mathrm{SM}} \mathrm{BR}^{\mathrm{SM}}(h \rightarrow f)}
$$

where $\sigma$ is the SM-like Higgs production cross section and $\mathrm{BR}(h \rightarrow f)$ is the branching ratio of the decay into final state $f ; \sigma^{\mathrm{SM}}$ and $\mathrm{BR}^{\mathrm{SM}}(h \rightarrow f)$ are the theoretical values calculated for the same quantities in the SM. We will also make some rough prediction for the surviving 2HDM parameter space after the $13 / 14 \mathrm{TeV}$ run. To perform this study we will demand $\mu_{f}^{h}$ to be within 20,10 or $5 \%$ of the SM predictions for the final states $f=W W, Z Z, \gamma \gamma$ and $\tau^{+} \tau^{-}$. Finally we define the constants $\kappa_{i}$ as

$$
\kappa_{i}^{2}=\frac{\Gamma^{2 \mathrm{HDM}}(h \rightarrow i)}{\Gamma^{\mathrm{SM}}(h \rightarrow i)},
$$

which at tree-level are just the $2 \mathrm{HDM}$ couplings relative to the SM $\kappa_{i}=g_{i}^{2 \mathrm{HDM}} / g_{i}^{\mathrm{SM}}$.

We will discuss two scenarios, one where the light CP-even Higgs is the SM-like one with $m_{h}=125.9 \mathrm{GeV}$ (the light Higgs scenario) and the other where the heavy CP-even scalar is the SM-like one with $m_{H}=125.9 \mathrm{GeV}$ (the heavy Higgs scenario). Therefore in our scans the mass of one of the CP-even states is set to $125.9 \mathrm{GeV}$. All other masses are free to run in the range $[50,1000] \mathrm{GeV}$, subject to all constraints plus the condition that the other neutral scalars are at least 

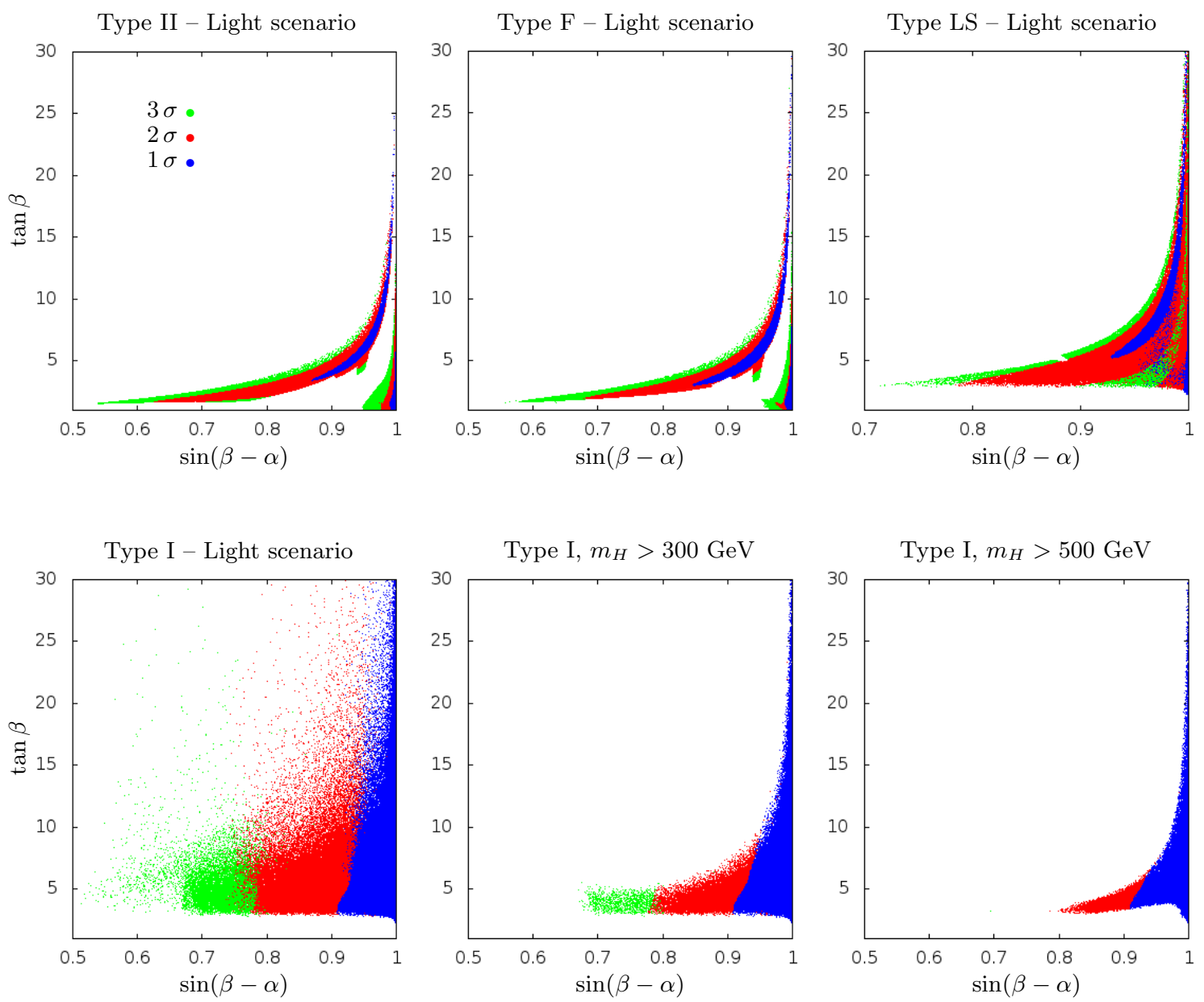

Figure 1: Light Higgs scenario: Allowed parameter space in the $\tan \beta$ vs $\sin (\beta-\alpha)$ plane after the LHC $8 \mathrm{TeV}$ run for the Light Higgs scenario for each model type. Points have been accepted according to their $\mathrm{p}$ value being within a number of standard deviations as show in the key (see top left panel). We have imposed that $m_{A}>m_{h}+5 \mathrm{GeV}$.

$5 \mathrm{GeV}$ away from the Higgs mass $125.9 \mathrm{GeV}$. Regarding the remaining parameters, they will be varied in the range $0.1<\tan \beta<50,|\alpha|<\pi / 2$ and $-(900 \mathrm{GeV})^{2}<m_{12}^{2}<(900 \mathrm{GeV})^{2}$.

In figure 1 we present the points that survived at $3 \sigma, 2 \sigma$ and $1 \sigma$ for the light Higgs scenario in the $\tan \beta$ vs $\sin (\beta-\alpha)$ plane, with all other constraints applied. Type II and $\mathrm{F}$ are shown in the top left and middle panels. The allowed parameter space is clearly centred around the lines $\sin (\beta-\alpha)=1$, the SM-like or alignment limit, and $\sin (\beta+\alpha)=1$ which, for type II and $\mathrm{F}$ is the wrong sign limit introduced in [23], and discussed again in detail in [14] (see also [24]). The models have very similar allowed regions. These regions are primarily related to the bound on the $\mu_{V V}$ as pointed out in [25] and discussed in great in detail in [24]. In fact, as shown in [24], it is enough to impose $0.8<\mu_{V V}<1.2$ to reproduce figure 1 for type II and $\mathrm{F}$ with remarkable accuracy.

The three bottom figures refer to the type I model. Again, the shape of the allowed region is to a great extent determined by $\mu_{V V}$ which in type I is approximately equal to $\sin ^{2}(\beta-\alpha)$ [25]. This 
is clearly seen in the bottom left panel because the limits are almost vertical lines and therefore independent of $\tan \beta$. This is due to the fact that if $\tan \beta \gg 1$ the type I $2 \mathrm{HDM}$ is equivalent to the model obtained by adding a singlet to the SM. In fact, in this limit $\kappa_{F} \approx \kappa_{V}=\sin (\beta-\alpha)$ and only small deviations occur for small $\tan \beta$. The effect of the self-couplings can only be seen via the introduction of the theoretical constraints which is shown in the middle and right bottom plots. However, the effect only occurs with the introduction of a lower bound on $m_{H}$. The combination of theoretical constraints with a $300 \mathrm{GeV}$ (middle) and $500 \mathrm{GeV}$ (right) lower bound has a remarkable effect on the allowed parameter space of the type I model. This behaviour is mainly driven by the constraint that forces the minimum to be the global one at tree-level [13].

Finally, in the top right plot we present the allowed parameter space for type LS. As discussed in [14] it reflects the fact that $\mu_{V V}$ is now somehow between type I and type II. Depending on the values of $\tan \beta$ and $\sin (\beta-\alpha), \mu_{V V}$ is closer to type II (when $h \rightarrow \tau^{+} \tau^{-}$dominates) or closer to type I (when $h \rightarrow b \bar{b}$ dominates). In the type LS model the measurement of $\mu_{\tau \tau}$ has an important effect on the parameter space of the model [26] especially for large $\tan \beta$.

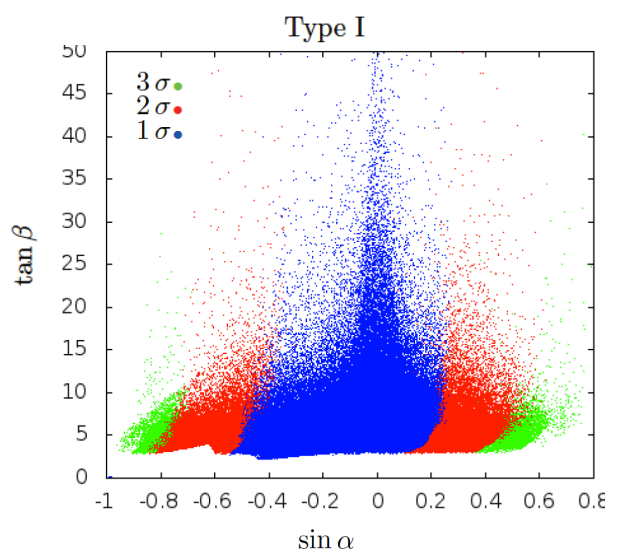

Figure 2: Light Higgs scenario: The fermiophobic limit in type I $2 \mathrm{HDM}(\cos \alpha=0)$ is excluded at more than $3 \sigma$ after the LHC run-1.

The type I model has a limit where the lightest Higgs completely decouples from the fermions. This is called the fermiophobic limit and is obtained by setting $\sin \alpha= \pm 1$ [27]. In figure 2 we present the allowed parameter space for type I in the plane $(\tan \beta, \sin \alpha)$. Clearly, one can state that the fermiophobic limit of a type I $2 \mathrm{HDM}$ is now excluded at $3 \sigma$.

\subsection{The future of CP-conserving 2HDM}

We will now analyse the impact on the $2 \mathrm{HDM}$ parameter space of an increase in the accuracy of the rates' measurement from the present values of about $20 \%$ to $10 \%$ or even $5 \%$ relative to the SM prediction. (see [28] for an analysis based on predictions for future colliders). We start with the wrong sign limit which is defined by $\kappa_{i} \kappa_{j}<0$ where $i, j$ can be either a fermion, $F$ or a gauge boson, $V$. According to our choice of the range of variation for $\alpha, \kappa_{U}$ is always positive. Furthermore, the interference of lepton loops with any other particle loops is always negligible for the expected accuracy. Finally, as shown in [14], the combination of all constraints disallows any wrong sign limit for $\tan \beta<1$. Therefore the only possible wrong sign limit, corresponding to the 

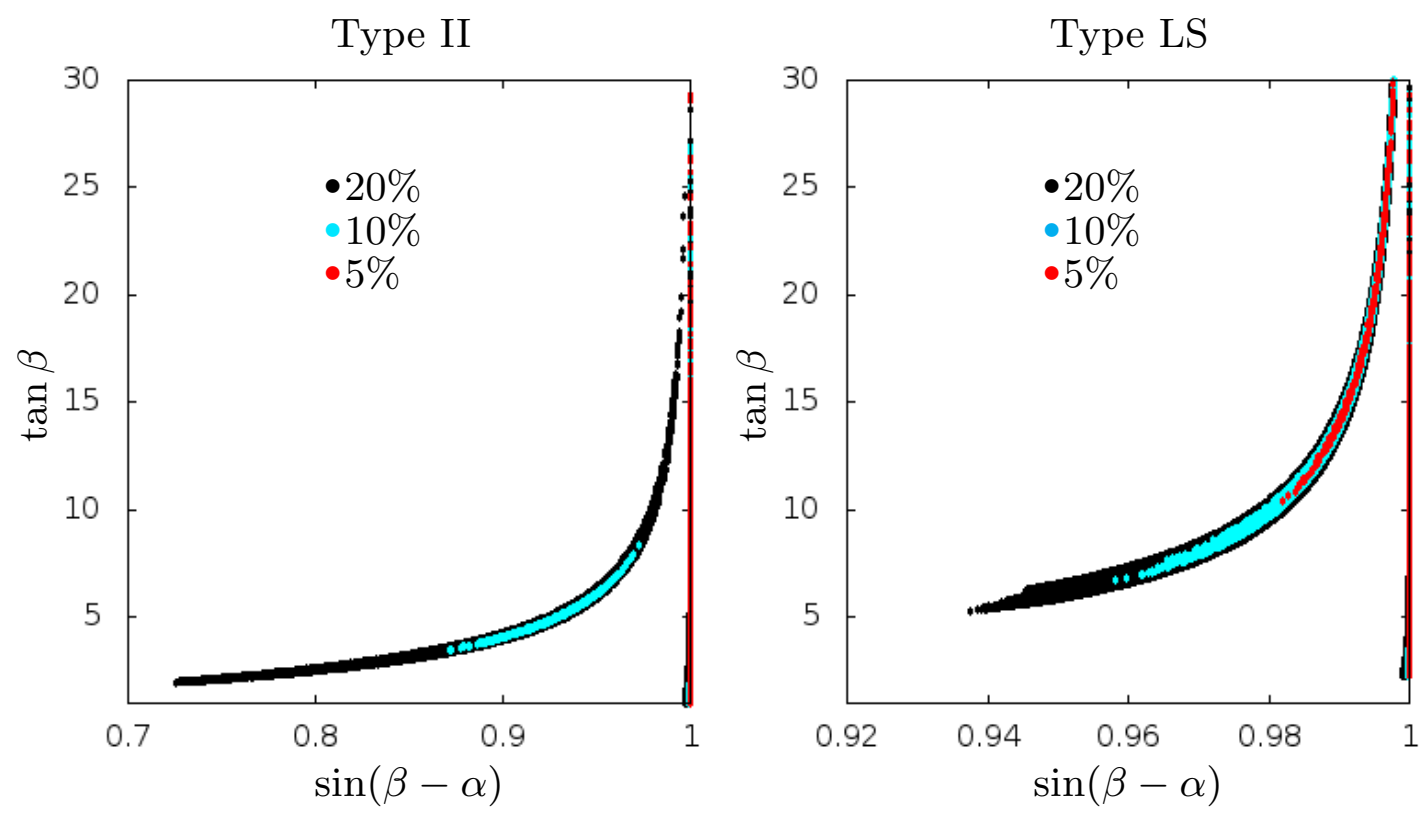

Figure 3: Light Higgs scenario: Predicted allowed parameter space in the $(\tan \beta, \sin (\beta-\alpha))$ plane for type II (left) and type LS (right) with rates measured at $20 \%$ (black), $10 \%$ (cyan) and $5 \%$ (red) accuracy.

left leg in the left panel of figure 3 , is $\kappa_{D} \kappa_{V}<0$ for type II and type F. At $20 \%$ we obtain similar results to the ones shown in figure 1 . Already at $10 \%$ the allowed space for the wrong sign limit is reduced due to $\mu_{\tau \tau}$. This should however be interpreted with care due to the the uncertainties in the $g g$ production cross section. Finally, as discussed in [23] this wrong sign limit is excluded if $\mu_{\gamma \gamma}$ does not deviate by more than 5\% from the SM prediction. The exclusion is mainly due to the contribution of the charged Higgs loop to $h \rightarrow \gamma \gamma$. As shown in [24] this result is independent of the value of the production cross section (provided the constraint on $\mu_{V V}$ is already taken into account).

There is no wrong sign limit for models type I and type LS when $\sin (\beta+\alpha)=1$ (and $\tan \beta>1$ due to experimental constraints) [23, 14]. However, the right panel of figure 3 clearly shows a left leg very similar to the one obtained for types II and F. The main difference lies in the range of allowed values of $\sin (\beta-\alpha)$ in the two cases. What is the origin of the second leg in type LS? When $\sin (\beta+\alpha)=1$ (which corresponds to $\alpha \rightarrow-\alpha$ relative to alignment) we have

$$
\kappa_{V}=\frac{\tan ^{2} \beta-1}{\tan ^{2} \beta+1}
$$

and since $\tan \beta \gtrsim 1, \kappa_{V}>0$. Therefore, in types LS (and in type I) we obtain $\kappa_{U}=\kappa_{D}=1$ and the only difference relative to the alignment limit is that $\kappa_{V} \neq 1$, except for very large $\tan \beta$. The limit where $\sin (\beta+\alpha)=1$ but no sign change occurs in any of the Higgs couplings to the remaining SM particle was defined as the symmetric limit [14]. Equation 4.3 tells us that for low $\tan \beta$ there are good chances of distinguishing the symmetric limit from the alignment limit which is clearly seen in the figure. However, it also clear that for $\tan \beta$ above $\approx 10$ the two limits become indistinguishable even with a $5 \%$ accuracy in the measured rates. 


\subsection{Non-decoupling in the heavy scenario}

In the previous section we have presented the status of the 2HDM in the light Higgs scenario. The scenario where the heavy CP-even scalar is the SM one can be obtained from the light one with the mapping (which preserves our convention),

$$
\alpha \rightarrow \alpha-\operatorname{sign}(\alpha) \frac{\pi}{2} .
$$

and a detailed discussion regarding the heavy scenario can be found in [14]. There is however a major difference between the two scenarios. While a decoupling limit exists for the light scenario of the 2HDM [29], the same is not true for the heavy scenario. In fact, because $m_{h}<m_{H}=125.9$ $\mathrm{GeV}$, the heavy scenario has an alignment limit when $\sin (\beta-\alpha)=0$ but not a decoupling limit. In figure 4 we show in the left panel $\kappa_{\gamma}$ as a function of $\kappa_{U}$ for the heavy scenario. As expected,
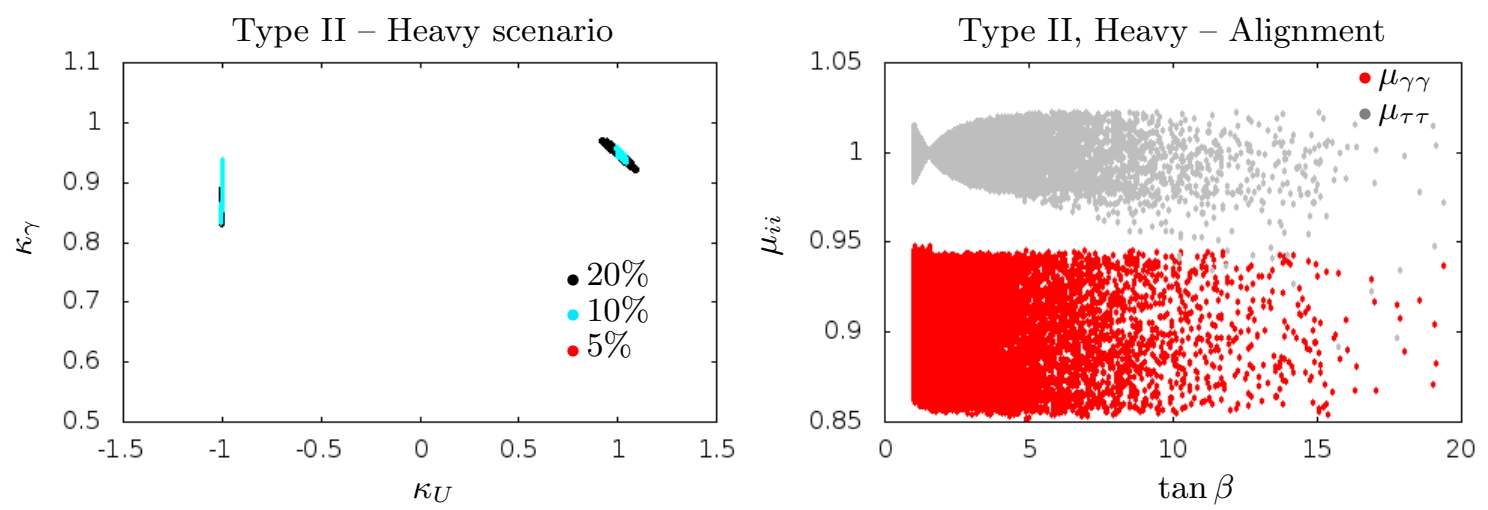

Figure 4: Heavy Higgs scenario: in the left panel we show $\kappa_{\gamma}$ as a function of $\kappa_{U}$ with rates measured at 20 $\%$ (black), $10 \%$ (cyan) and $5 \%$ (red) accuracy; in the right panel we have $\mu_{\gamma \gamma}$ and $\mu_{\tau \tau}$ as a function of $\tan \beta$ with all theoretical and pre-LHC experimental constraints plus $\mu_{V V}$ measured with $5 \%$ accuracy.

the wrong sign limit is excluded with a 5\% accuracy measurement of the rates. This is exactly what happens for the light scenario and is due to $\mu_{\gamma \gamma}$ as previously discussed. What is new for the heavy case is that also the alignment limit can be excluded with the same accuracy. The right panel of figure 4 shows that $\mu_{\gamma \gamma}$ is always below 0.95 . The reason for this major difference lies in the charged Higgs loop contribution when combined with the theoretical constraints. The charged Higgs couplings to the heavy CP-even Higgs have the form (for $\tan \beta>1$ )

$$
g_{H H^{ \pm} H^{\mp}}^{\text {Wronsign }}=-\frac{2 m_{H^{ \pm}}^{2}-m_{H}^{2}}{v^{2}}, \quad g_{H H^{ \pm} H^{\mp}}^{\text {Alignmen }}=-\frac{2 m_{H^{ \pm}}^{2}+m_{H}^{2}-2 M^{2}}{v^{2}}
$$

for the wrong sign and alignment limit respectively. In figure 5 we show the allowed range of the pre-factor that multiplies the charged Higgs loop contribution to $\Gamma(h \rightarrow \gamma \gamma)$ for the alignment limit (left panel) and wrong sign limit (right panel), with all theoretical and pre-LHC experimental constraints plus $\mu_{V V}$ measured with $5 \%$ accuracy. The plots clearly show that the reduction in $H \rightarrow \gamma \gamma$ has the same origin in both limits and is related to the negative and almost constant values of the charged Higgs loop pre-factor. We just need to understand why the the difference between 

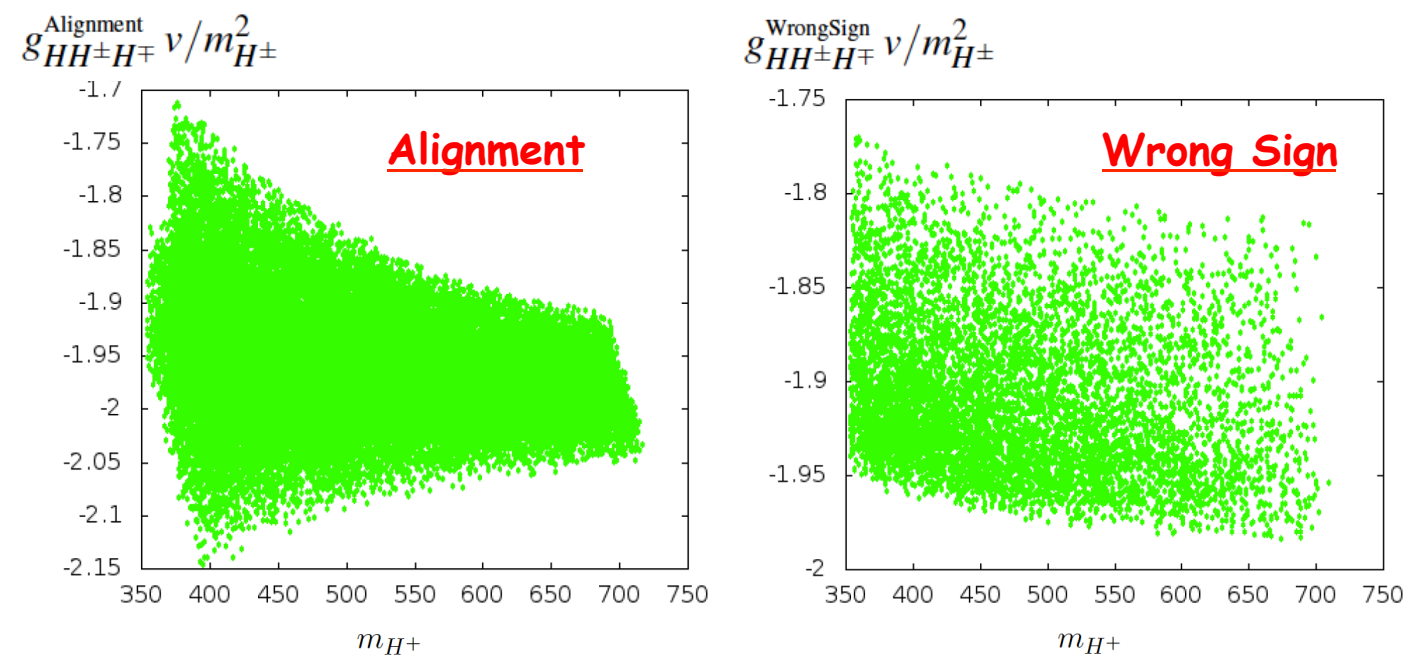

Figure 5: Heavy Higgs scenario: Allowed range for $g_{H H^{ \pm} H^{\mp}} v / m_{H^{ \pm}}^{2}$ for the alignment limit (left) and wrong sign limit (right), with all theoretical and pre-LHC experimental constraints plus $\mu_{V V}$ measured with $5 \%$ accuracy.

the two expressions in (4.5), which amounts to $2\left(m_{H}^{2}-M^{2}\right)$, is negligible. The same is to ask why is $m_{H} \backsim|M|$ and consequently small when compared to the charged Higgs mass in type II $\left(m_{H^{ \pm}}>340 \mathrm{GeV}\right.$, due to $\left.b \rightarrow s \gamma\right)$. In the alignment limit the boundedness from below condition $\lambda_{1}>0$, can be written as $M^{2}<m_{H}^{2}+m_{h}^{2} / \tan ^{2} \beta$ which shows that indeed $m_{H}^{2} \backsim M^{2} \ll m_{H^{ \pm}}^{2}$. One final remark is in order - it is the global minimum condition that forces $M^{2}>0$ and points in the 2HDM parameter space with $\mu_{\gamma \gamma}$ above 0.95 are allowed if all theoretical constraints are removed.

\section{Other versions of the $2 \mathrm{HDM}$}

After the discovery of the Higgs boson at CERN many papers discussing the parameter space of the CP-conserving 2HDM have appeared (see [14] for references). As discussed in the introduction, there are other versions of the 2HDM that have also been analysed in view of the latest experimental results. The BGL models have been analysed in [30] while models that parametrize all flavour conserving versions in one model such as the aligned model were studied in [31] (we again note that this last class of models is not stable under radiative corrections). Finally, the parameter space of the explicit CP-violating 2HDM was analysed in [32].

\section{References}

[1] G. Aad et al. [ATLAS Collaboration], Phys. Lett. B 716, 1 (2012).

[2] S. Chatrchyan et al. [CMS Collaboration], Phys. Lett. B 716, 30 (2012).

[3] J.F. Gunion, H.E. Haber, G.L. Kane and S. Dawson, The Higgs Hunter's Guide (Westview Press, Boulder, CO, 2000).

[4] G.C. Branco, P.M. Ferreira, L. Lavoura, M.N. Rebelo, M. Sher and J.P. Silva, Phys. Rept. 516, 1 (2012). 
[5] S.L. Glashow and S. Weinberg, Phys. Rev. D 15, 1958 (1977); E.A. Paschos, Phys. Rev. D 15, 1966 (1977).

[6] V. D. Barger, J. L. Hewett and R. J. N. Phillips, Phys. Rev. D 41, 3421 (1990).

[7] P. M. Ferreira, L. Lavoura and J. P. Silva, Phys. Lett. B 688 (2010) 341.

[8] G. C. Branco, W. Grimus and L. Lavoura, Phys. Lett. B 380 (1996) 119.

[9] T. P. Cheng and M. Sher, Phys. Rev. D 35 (1987) 3484.

[10] A. Pich and P. Tuzon, Phys. Rev. D 80 (2009) 091702.

[11] I. F. Ginzburg, M. Krawczyk and P. Osland, hep-ph/0211371.

[12] P.M. Ferreira, R. Santos and A. Barroso, Phys. Lett. B 603 (2004) 219 [Erratum-ibid. B 629 (2005) 114]; M. Maniatis, A. von Manteuffel, O. Nachtmann and F. Nagel, Eur. Phys. J. C 48, 805 (2006); I. P. Ivanov, Phys. Rev. D 75 (2007) 035001 [Erratum-ibid. D 76 (2007) 039902].

[13] A. Barroso, P. M. Ferreira, I. P. Ivanov and R. Santos, JHEP 1306 (2013) 045.

[14] P. M. Ferreira, R. Guedes, M. O. P. Sampaio and R. Santos, JHEP 1412 (2014) 067.

[15] R. Coimbra, M. O. P. Sampaio and R. Santos, Eur. Phys. J. C 73 (2013) 2428.

[16] R. Costa, R. Guedes, M. O. P. Sampaio, and R. Santos, ScannerS project, October, 2014. http://scanners.hepforge.org.

[17] R. V. Harlander, S. Liebler and H. Mantler, Computer Physics Communications 184 (2013) 1605.

[18] A. Djouadi, J. Kalinowski and M. Spira, Comput. Phys. Commun. 108 (1998) 56.

[19] R. Harlander, M. Mühlleitner, J. Rathsman, M. Spira and O. Stål, arXiv:1312.5571 [hep-ph].

[20] https://twiki.cern.ch/twiki/bin/view/LHCPhysics/Cross.Sections

[21] P. Bechtle, O. Brein, S. Heinemeyer, O. Stål, T. Stefaniak, G. Weiglein and K. E. Williams, Eur. Phys. J. C 74 (2014) 2693.

[22] P. Bechtle, S. Heinemeyer, O. Stål, T. Stefaniak and G. Weiglein, Eur. Phys. J. C 74 (2014) 2711.

[23] P. M. Ferreira, J. F. Gunion, H. E. Haber and R. Santos, Phys. Rev. D 89 (2014) 115003.

[24] D. Fontes, J. C. Romão and J. P. Silva, Phys. Rev. D 90 (2014) 015021.

[25] P. M. Ferreira, R. Santos, H. E. Haber and J. P. Silva, Phys. Rev. D 87 (2013) 5, 055009.

[26] A. Arhrib, C. W. Chiang, D. K. Ghosh and R. Santos, Phys. Rev. D 85 (2012) 115003.

[27] V. D. Barger, N. G. Deshpande, J. L. Hewett and T. G. Rizzo, In*Argonne 1993, Physics at current accelerators and supercolliders* 437-442 [hep-ph/9211234]. L. Brucher and R. Santos, Eur. Phys. J. C 12 (2000) 87.

[28] S. Kanemura, K. Tsumura, K. Yagyu and H. Yokoya, Phys. Rev. D 90 (2014) 7, 075001.

[29] J. F. Gunion and H. E. Haber, Phys. Rev. D 67 (2003) 075019.

[30] F. J. Botella, G. C. Branco, A. Carmona, M. Nebot, L. Pedro and M. N. Rebelo, JHEP 1407 (2014) 078; G. Bhattacharyya, D. Das and A. Kundu, Phys. Rev. D 89 (2014) 095029.

[31] A. Celis, V. Ilisie and A. Pich, JHEP 1312 (2013) 095; D. López-Val, T. Plehn and M. Rauch, JHEP 1310 (2013) 134.

[32] D. Fontes, J. C. Romão and J. P. Silva, JHEP 1412 (2014) 043. 